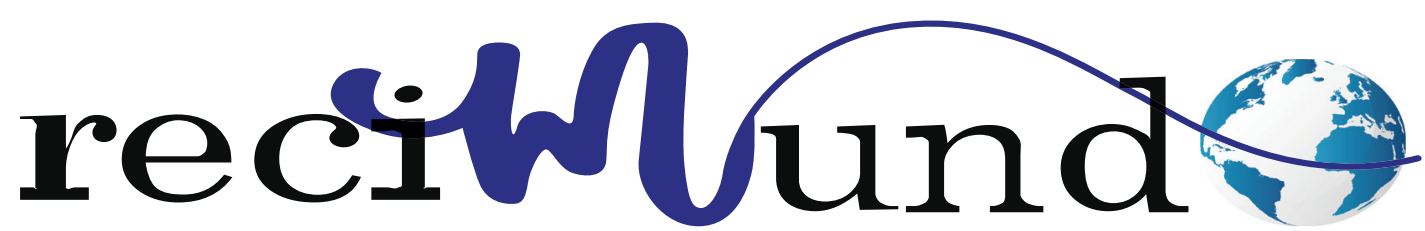

Revista Científica Mundo de la Investigación y el Conocimiento

DOI: 10.26820/recimundo/4.(2).mayo.2020.127-133

URL: http://recimundo.com/index.php/es/article/view/830

EDITORIAL: Saberes del Conocimiento

REVISTA: RECIMUNDO

ISSN: 2588-073X

TIPO DE INVESTIGACIÓN: Artículo de Revisión

Código UNESCO: 32 Ciencias Médicas; 3201 Ciencias Clínicas

PAGINAS: $127-133$

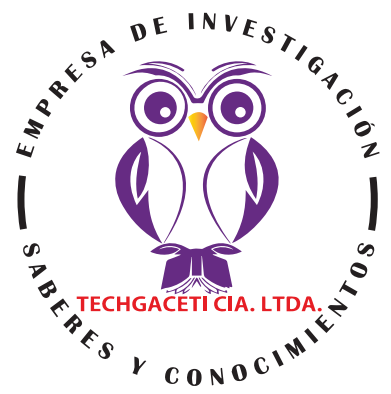

\title{
Onicomicosis por Trichophyton rubrum: presentación de un caso clínico
}

Onychomycosis caused by Trichophyton rubrum: presentation of a clinical case

Onicomicose causada por Trichophyton rubrum: apresentação de um caso clínico Juan Fernando Pupiales Paucar1; Susana Margoth López Chipantasi2;

Mary del Cisne Guerrero Guamán 3 ; Cristina Elizabeth Guamán Morales ${ }^{4}$

\section{RECIBIDO: 15/03/2020 ACEPTADO: 20/03/2020 PUBLICADO: 20/05/2020}

1. Médico General de la Universidad Central del Ecuador; Quito, Ecuador; jfpp2611@gmail.com; (D) https://orcid. org/0000-0002-1822-9271

2. Médico General de la Universidad Central del Ecuador; Quito, Ecuador; susanamargothlopez@gmail.com; (D) https://orcid.org/0000-0002-7945-8772

3. Médico General de la Universidad Central del Ecuador; Quito, Ecuador; marycisne.guerrero@gmail.com; https://orcid.org/0000-0002-7162-7906

4. Médico General de la Universidad Central del Ecuador; Quito, Ecuador; cristina0106gm@gmail.com; (DD https://orcid.org/0000-0003-3184-1606

CORRESPONDENCIA

Juan Fernando Pupiales Paucar

jfpp2611@gmail.com

Quito, Ecuador

○ RECIMUNDO; Editorial Saberes del Conocimiento, 2020 


\section{RESUMEN}

La onicomicosis es la infección micótica de las uñas causada por dermatofitos o levaduras. Su importancia radica en los problemas asociados como dolor, parestesias, dificultades al realizar actividades de la vida diaria y perjudicar las interacciones sociales, por eso es importante indicar un adecuado tratamiento. En el presente artículo se revisa la respuesta al tratamiento con 2 anti fúngicos orales (fluconazol y terbinafina). Se presenta el caso clínico de un paciente de sexo masculino de 24 años que presenta una lesión hipertrófica, distrófica, amarillenta y descamativa en el primer dedo del pie izquierdo de 2 años de evolución con tratamiento previo de fluconazol 300mg una vez a la semana durante 1 año sin tener la respuesta esperada el cual acude al Centro de salud El Chaupi en donde después de exámenes diagnósticos con Hidróxido de Potasio y Cultivo de hongos se indica tratamiento con terbinafina 250 mg una vez al día por 4 meses más empleo de ciclopirox al $8 \%$.

Palabras clave: Onicomicosis, dermatofitos, terbinafina, fluconazole.

\section{ABSTRACT}

Onychomycosis is the fungal infection of the nails caused by dermatophytes or yeasts. Its importance lies in the associated problems such as pain, paraesthesia, difficulties in performing activities of daily living and damaging social interactions, so it is important to indicate adequate treatment. This article reviews the response to treatment with 2 oral antifungals (fluconazole and terbinafine). We here report a clinical case of an a male patient of a 24-year-old with 2 years history of a hypertrophic, dystrophic, yellowish and scaly lesion on the first left toe with prior treatment of fluconazole 300mg once a week for 1 year without having the expected response which goes to the El Chaupi Health Center where after diagnostic tests with Potassium Hydroxide and Mushroom Cultivation, The patient was treated with terbinafine $250 \mathrm{mg}$ once a day for 4 months plus use of cyclopirox at $8 \%$.

Keywords: Onychomycosis, dermatophytes, terbinafine, fluconazol.

\section{RESUMO}

Onicomicose é a infecção fúngica das unhas causada por dermatófitos ou leveduras. Sua importância está nos problemas associados, como dor, parestesia, dificuldades na realização de atividades da vida diária e interações sociais prejudiciais, sendo importante indicar tratamento adequado. Este artigo analisa a resposta ao tratamento com 2 antifúngicos orais (fluconazol e terbinafina). Relatamos aqui um caso clínico de um paciente do sexo masculino, 24 anos, com 2 anos de história de lesão hipertrófica, distrófica, amarelada e escamosa no primeiro dedo do pé esquerdo com tratamento prévio de fluconazol 300mg uma vez por semana durante 1 ano sem tendo a resposta esperada que vai ao Centro de Saúde El Chaupi, onde após testes diagnósticos com Hidróxido de Potássio e Cultivo de Cogumelos, o paciente foi tratado com terbinafina 250 mg uma vez ao dia por 4 meses mais uso de ciclopirox a 8\%.

Palavras-chave: Onicomicose, dermatófitos, terbinafina, fluconazol. 


\section{Introducción}

La onicomicosis es la infección ungueal que se presenta con mayor frecuencia, representa del 18 al $50 \%$ de todas las infecciones fúngicas. En general se estima una prevalencia del 3 al 13,8\%. Estos valores pueden incrementar en pacientes con factores de riesgo, como: tiña de pies, uso de calzado estrecho, actividades deportivas (natación), uso de baños comunitarios o públicos, traumatismos, edad avanzada, comorbilidades asociadas, inmunodeficiencia y predisposición genética, etc [1].

Generalmente la onicomicosis inicia después de una tiña crónica de los pies, en donde las esporas de los hongos infecta el borde libre de las uñas, sin embargo puede producirse por otros mecanismos. Es causada por una gran variedad de microorganismos, con mayor prevalencia de dermatofitos con predominio de Trichophyton rubrum y T. mentagropbytes; los hongos levaduriformes (levaduras), particularmente del género Cándida siendo la más frecuente C. albicans y C. parapsilosis; finalmente puede deberse también a Aspergillus y Scopulariopsis brevicaulis.

Existen varias formas clínicas entre ellas: onicomicosis primaria en la que la invasión fúngica afecta la uña intacta mientras que la secundaria produce daño en una uña antes afectada por enfermedades o traumas [2]. Además existen la onicomicosis subungueal distal y lateral, subungueal proximal, distrófica total y blanca superficial. Se sugiere agregar la onicomicosis de patrón mixto siendo aquella infección en donde existen dos o más formas clínicas en el mismo paciente (incluso en la misma uña); siendo más común la asociación: subungueal proximal con superficial y subungueal lateral/distal con superficial; pudiéndose encontrar un solo agente causal o varios, los más frecuentes son T. rubrum y fusarium. La forma secundaria que se presenta debido a otras patologías no fúngicas pudiendo de- berse a traumatismos, o psoriasis. Debido a la similitud clínica y la dificultad para el diagnóstico es esencial realizar prueba micológicas que confirmen la presencia del agente etiológico [3].

Los hongos de moho y los dermatofitos presentan clínica similar, producen paquioniquia, pulverización distal, xantoniquia y otros cambios de coloración de la lámina ungueal. En los casos producidos por levaduras como Cándida se caracteriza la presencia de paroniquia o peronixis (inflamación periungueal), siempre acompaña de dolor [4].

Los tratamientos suelen ser de larga duración debido al crecimiento lento de las uñas y debido a esto no suelen observarse resultados satisfactorios inmediatos por parte del paciente, de igual manera las tasas bajas de curación suelen ir asociadas a comorbilidades y a diagnósticos micológicos incorrectos. Por otro lado, es importante considerar los problemas farmacológicos debido a la capacidad de penetración y el porcentaje de retención del anti fúngico en la uña y la existencia de resistencias primarias y secundarias [1].

Los cambios que ha experimentado el tratamiento ha pasado del uso de preparaciones locales al uso de nuevos agentes anti fúngicos, de uso tópico u oral, sin embargo es fundamental la identificación del agente causal para conseguir una buena indicación terapéutica por medio de la confirmación en el laboratorio, lo que no se realiza en todos los casos [4].

\section{Caso Clínico}

Se presenta el caso clínico de un paciente de sexo masculino de 24 años de edad nacido y residente en la ciudad de Quito, ocupación estudiante, como antecedentes patológicos personales, no refiere patología, como antecedentes patológicos familiares, abuela paterna con antecedente de

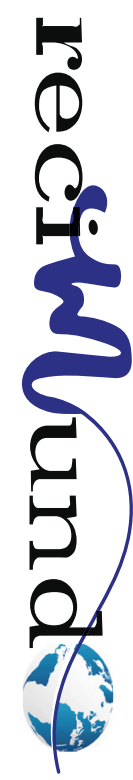


demencia senil. Acude al servicio de medicina general del Centro de Salud El Chaupi de la Ciudad de Machachi por presentar una lesión hipertrófica, distrófica, amarillenta y descamativa a nivel del primer dedo del pie izquierdo de 2 años de evolución, refiere haber recibido tratamiento con fluconazol 300 mg vía oral 1 vez a la semana durante un año, manifiesta que el medicamento prescrito no logro curar la lesión hipertrófica y distrofica a nivel del primer dedo del pie izquierdo. Debido a que las lesiones persistían acude al servicio de medicina general quien solicita la realización de un examen directo con Hidróxido de Potasio $(\mathrm{KOH})$ y un cultivo de hongos, posteriormente en el laboratorio clínico se toman muestras de escamas del primer dedo del pie izquierdo (Fig. 1a) (Fig. 1b), el resultado del $\mathrm{KOH}$ presento hifas fuliginosas septadas, en el cultivo de hongos se identifica Trichophyton rubrum. Posteriormente se inicia tratamiento con terbinafina $250 \mathrm{mg}$ vía oral diaria por 4 meses más ciclopirox al 8\% laca que se debe aplicar sobre la uña afectada una vez al día previo lavado y secado de la uña, tras concluir los 4 meses de tratamiento se solicita exámenes de laboratorio para valorar la función hepática del paciente y cuyos resultados fueron GAMA GT $21 \mathrm{U} / \mathrm{L}$, AST 18.3 $\mathrm{U} / \mathrm{L}, \mathrm{ALT} 18.1 \mathrm{U} / \mathrm{L}$, al no encontrar valores alterados y al encontrar una evolución favorable de la lesión (Fig. 2a) (Fig. 2b) se decide continuar el tratamiento con terbinafina durante 2 meses. Al final del tratamiento se logra evidenciar una placa ungueal libre de hipertrofia y distrofia (Fig. 3a) (Fig. 3b) (Fig. 4a) posteriormente se solicita un nuevo examen $\mathrm{KOH}$ cuyo resultado es negativo y un cultivo de hongos cuyo resultado es negativo.

\section{Discusión}

Durante años la onicomicosis se consideró como un problema estético, sin embargo, con el pasar de los años se ha ido cambiando este concepto, más que un problema estético representa un trastorno medico importante que conlleva consecuencias físicas, fisiológicas, psicosociales y ocupacionales, afectando la salud física, social y emocional [5].

La onicomicosis es causada comúnmente por dermatofitos seguidos de mohos no dermatofitos y levaduras, el diagnóstico puede sospecharse por los aspectos clínicos del paciente aunque es indispensable la confirmación de laboratorio antes del tratamiento [6]. En el presente caso clínico se observa que el paciente ya tenía un tratamiento previo fallido sin la realización de ningún examen diagnóstico previo, por lo que fue indispensable la realización de la prueba de hidróxido de potasio y el cultivo de hongos (el mismo que identifico a Trichophyton rubrum como el agente causal) y así poder iniciar un tratamiento adecuado para el paciente evitando un nuevo fallo en el tratamiento.

El tratamiento de elección para la onicomicosis debe ser el que cuente con una penetración efectiva de las uñas y una actividad anti fúngica de amplio espectro dentro de la uña, con una dosis conveniente y que tenga escasos efectos secundarios. El adecuado tratamiento debe tener como objetivo tasas generales de curación y mayor efectividad [7].

Actualmente existen varias formas de tratar la onicomicosis incluyendo terapias antimicóticas orales, terapias antimicóticas tópicas y terapias basadas en dispositivos, solas o en combinación. El tratamiento antimicótico oral se usa para enfermedad moderada a grave o cuando múltiples uñas se ven afectadas. Por otro lado, el tratamiento tópico puede ser considerado para enfermedad leve a moderada, aunque la evidencia científica manifiesta que el tratamiento combinado (entre la terapia oral y tópica) aumenta la tasa de cura de la enfermedad [6]. El tratamiento de elección es la terbinafina que es un agente fungicida del grupo alilamina con escasos efectos secundarios. 
Por otro lado hay otros medicamentos como el itraconazol y fluconazol que son fungistáticos y tienen más efectos secundarios e interacciones farmacológicas. La dosis para un paciente adulto de terbinafina es de 250 mg vía oral cada día por 3 a 4 meses [6].

En relación a la terapia tópica implica el uso de lacas de uñas las mismas que se aplican en la cara dorsal de la uña, los agentes anti fúngicos tópicos comúnmente utilizados son efinaconazol (Jublia, Clenafin) al 10\%, ciclopirox (Oni-tec) al 8\%, amorolfina (Curanail, Loceryl, Locetar, Odenil) al $5 \%$ y terbinafina (Lamisil) al 10\%. Cabe mencionar que el tratamiento tópico es ineficaz en Onicomicosis con lesiones avanzadas y graves [6].

En el presente caso clínico el paciente ya había presentado un tratamiento previo con Fluconazol 300 mg vía oral 1 vez a la semana por 1 año el mismo que fue fallido ya que no se obtuvo ninguna cura, por lo que basados en la evidencia científica y con él la identificación del agente causal se decide iniciar el tratamiento de elección con terbinafina 250 mg vía oral cada día por 4 meses acompañado del tratamiento tópico con ciclopirox al 8\% que se aplica $1 \mathrm{vez}$ al día previo al uso de medidas higiénicas sobre la uña.
Finalmente queda demostrado que el medicamento de elección en la actualidad es la terbinafina en conjunto con un tratamiento tópico aumentando así la tasa de curación, se recalca que el tratamiento se lo debe realizar lo antes posible para evitar el contagio a uñas de los otros dedos, además es indispensable mantener medidas higiénicas adecuadas para evitar la reinfección como son; el lavado y secado adecuado de los pies, el corte adecuado de las uñas que no produzcan lesiones sobre el tejido ungueal, el cambio diario de calcetines y el uso de calzado limpio que no produzca sudoración de los pies y que no haga presión sobre los mismos con el fin de evitar el crecimiento de los distintos tipos de hongos que pueden causar infección [6].

\section{Conclusión}

Se concluye que el tratamiento de primera línea para la onicomicosis es la terbinafina siendo causada con mayor frecuencia por Trichopyton rubrum.

Además se determina la importancia de la realización de exámenes complementarios para realizar una terapéutica adecuada y evitar resistencia farmacológica.

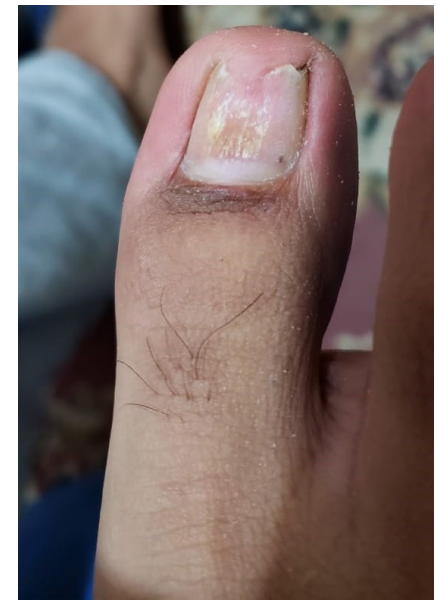

Figura 1a. Lesión onicodistrofica

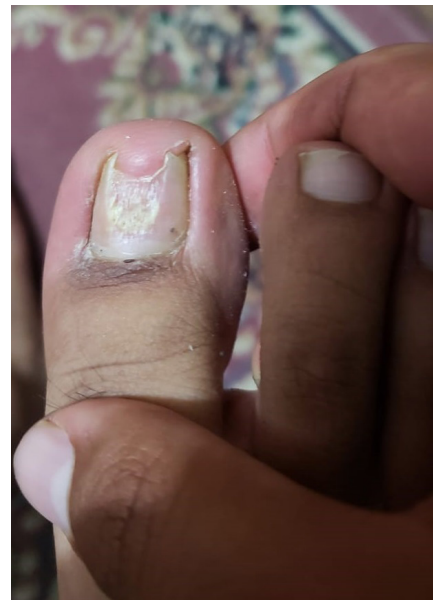

Figura 1b. Lesión subungueal proximal

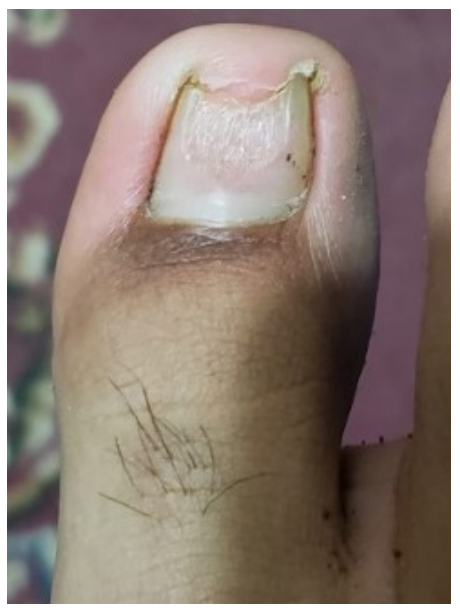

Figura 2 a. Lesion subungueal distal 2 meses de tratamiento

Fuente: Los autores 


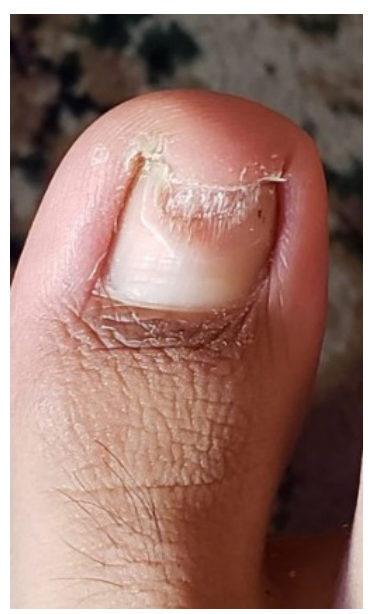

Figura 2b. Lesion subungueal distal 4 meses de tratamiento

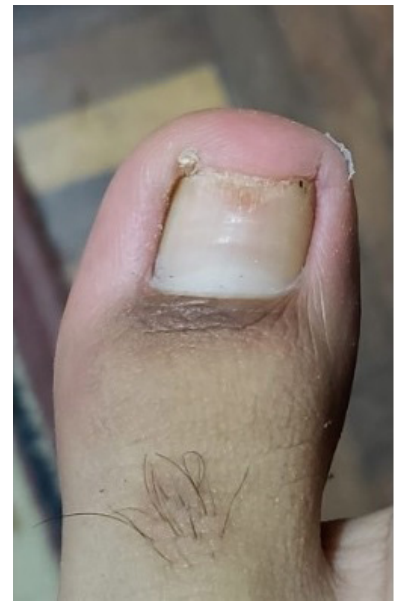

Figura 3a. Lesión subungueal distal 6 meses de tratamiento

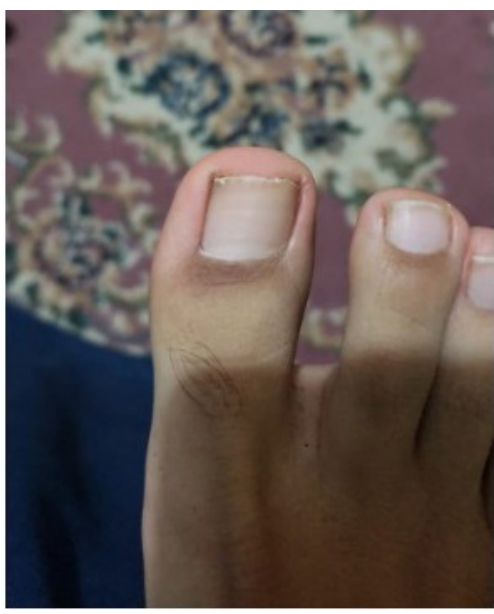

Figura 4a. Uña sana a los 9 meses

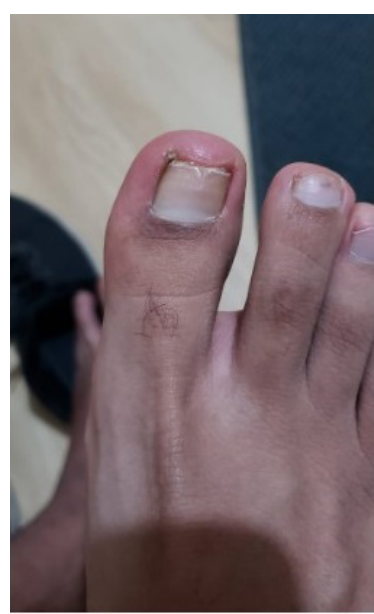

Figura 3b. Lesión subungueal a los 7 meses

Fuente: Los autores

\section{Conflicto de intereses}

Los autores declaran no tener conflictos de intereses.

\section{Financiamiento}

Los autores declaran que el presente trabajo fue autofinanciado.

\section{Bibliografía}

[1] Carrillo-Muñoz AJ, Tur-Tur C, Hernández-Molina $\mathrm{JM}$, et al. Antifúngicos disponibles para el tratamiento de las micosis ungueales. Rev Iberoam Micol 2010; 27: 49-56.

[2] Giniebra Marín GM, Rivera Rivadulla R, Linares Cánovas LP, et al. Onicomicosis, factores predis- ponentes, características y dermatosis asociadas. Ciencas Médicas Pinar del Río, http://www.revcmpinar.sld.cu/index.php/publicaciones/article/ view/3926/html (2019).

[3] Lipner SR, Scher RK. Onychomycosis. J Am Acad Dermatol 2019; 80: 853-867.

[4] Tchernev G, Penev PK, Nenoff P, et al. Ony- 
chomycosis: modern diagnostic and treatment approaches. Wiener Medizinische Wochenschrift 2013; 163: 1-12.

[5] Cobos Lladó D, Fierro Arias L, Arrellano Mendoza I, et al. La onicomicosis y su influencia en la calidad de vida. Dermatología Cosmética, Médica y Quirúrgica; 14, https://www.medigraphic.com/ pdfs/cosmetica/dcm-2016/dcm164h.pdf (2016).
[6] K.C. Leung A, M Lam J, Leong KF, et al. Onychomycosis: An Updated Review. Recent Pat Inflamm Allergy Drug Discov; 13. Epub ahead of print 26 October 2019. DOI: 10.2174/1872213X13 666191026090713.

[7] Kawa N, Lee KC, Anderson RR, et al. ONYCHOMYCOSIS: A Review of New and Emerging Topical and Device-based Treatments. J Clin Aesthet Dermatol 2019; 12: 29-34.

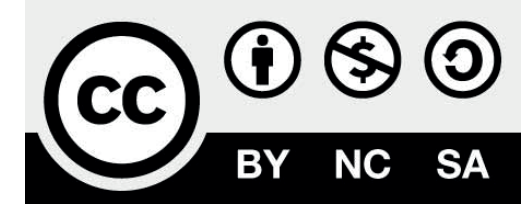

RECONOCIMIENTO-NOCOMERCIAL-COMPARTIRIGUAL CC BY-NC-SA

ESTA LICENCIA PERMITE A OTROS ENTREMEZCLAR, AJUSTAR Y CONSTRUIR A PARTIR DE SU OBRA CON FINES NO COMERCIALES, SIEMPRE $Y$ CUANDO LE RECONOZCAN LA AUTORÍA Y SUS NUEVAS CREACIONES ESTÉN BAJO UNA LICENCIA CON LOS MISMOS TÉRMINOS.

\section{CITAR ESTE ARTICULO:}

Pupiales Paucar, J., López Chipantasi, S., Guerrero Guamán, M., \& Guamán Morales, C. (2020). Onicomicosis por Trichophyton rubrum: presentación de un caso clínico. RECIMUNDO, 4(2), 122-129. doi:10.26820/recimundo/4.(2). mayo.2020.127-133 